\title{
Clases demostrativas interactivas para la formación de profesores en línea
}

\section{Interactive Demonstrative Lectures in online Teachers Training}

\author{
Mario Humberto Ramírez Díaz \\ Instituto Politécnico Nacional, Ciudad de México (México) \\ mramirezd@ipn.mx
}

\begin{abstract}
Resumen
La formación de profesores de física es una necesidad creciente dado el bajo nivel mostrado por los estudiantes en diversos indicadores tales como PISA. Sin embargo, el que los profesores puedan asistir de manera presencial a las universidades es difícil dada su carga académica frente a grupo. Por lo anterior, la formación en línea se ha convertido en una opción viable que permite no solo la preparación de los profesores sino además el intercambio con colegas más allá de su medio y región. Una característica que ha tenido la educación en línea es la de "adaptar" metodologías propias de los programas presenciales en sus estrategias de aprendizaje. En este trabajo mostramos los resultados de adaptar las Clases Demostrativas Interactivas en la formación de profesores de física que cursan estudios de Doctorado en Física Educativa. Se encuentra que la metodología permite no solo un aprendizaje efectivo en los profesores, sino que además produce un efecto multiplicador al permitir a los propios profesores al reproducir esta misma metodología con sus propios estudiantes. Finalmente, se muestra cómo se mejora la comunicación de experiencias por parte de los profesores al tener intercambio a distancia con colegas de diversos países participantes en el ejercicio.

Palabras Clave

Formación Docente, Física Educativa, Aprendizaje Activo, Educación a Distancia, Estudios de Posgrado.
\end{abstract}

\begin{abstract}
Physics teachers training is a growing necessity because the low level shown by students in several indicators like PISA. However, that teachers could go in presential form to universities is not easy because their hard work in class. For this the online formation has become in a viable option that allow not only the teachers training but interchange with colleagues in other beyond of their own region. On the other hand, a characteristic that have been the online education in "adapting" methodologies from presential education in its own learning strategies. In this work we show the results of adapting of Interactive Demonstrative Lectures of Sokoloff to physics teachers training that are made doctoral courses in physics education. We found that the methodology allows not only an effective learning to teachers, also produce a multiplier effect when the teachers reproduce this methodology with their students. Finally, we show how is better the experiences communication between the teachers when they chat online with colleagues in several countries in this exercise.
\end{abstract}

Keywords

Teachers Training, Physics Education, Active Learning, Online Education, Postgraduate Programs. 


\section{Introducción.}

La física es una de las ciencias que presentan una mayor dificultad en su comprensión por parte de los estudiantes, esta dificultad es evidente en el bajo índice de aprobación (alrededor del 30\% dependiendo la fuente) de esta disciplina en los diferentes niveles educativos en donde se imparte como lo muestran indicadores construidos por organismos internacionales como Proyecto Pisa, AAPT o Proyecto Tuning (Ramírez, Pérez-Trejo, Méndez-Sánchez y Olvera, 2016) entre otros. Los profesionales encargados de enseñar física en las escuelas difícilmente introducen metodologías novedosas en su práctica, tienen la tendencia de repetir la forma en la que fueron "instruidos" en la física, es decir, en forma tradicional, donde un experto da una clase magistral sin tener prácticamente interacción con los estudiantes. Estos dos aspectos: bajo índice de aprobación e instrucción tradicional, dan pie a cuestionar sobre la necesidad de incorporar elementos nuevos en la enseñanza de la física y sobre todo en la formación de profesores de física (Brisco, 1991, Bromme, 1988, Cronin-Jones, 1991).

En este sentido, el aprendizaje activo ha llevado a desarrollar metodologías para el mejoramiento del aprendizaje de la física, las llamadas Clases Demostrativas Interactivas (CDI) (Sokoloff, 2006). Las CDI tienen características generales tales cómo utilizar la experiencia concreta de los estudiantes para construir conceptos, utilizar ciclos de aprendizaje, intercambio y retroalimentación de las experiencias de los estudiantes, prueba de conceptos por medio de la experimentación, entre otros. Las CDI han sido probadas con éxito también en la región latinoamericana para diferentes temas de física como magnetismo, óptica o fuerza (Ramírez, Ortega y Ramírez, 2013, Sokoloff, 2006, Hernández, Tecpan y Osorio, 2015).

Por otro lado, la UNESCO ha apoyado el uso de metodologías de aprendizaje activo de la física especialmente porque promueven que el estudiante realice tareas experimentales que favorecen el aprendizaje conceptual, a la vez que se alienta al docente a practicar en el aula investigación en la enseñanza de la física (Sokoloff, 2006). Se utiliza la secuencia de aprendizaje predicción-discusión en pequeños grupos-observación de experimentos y comparación de los resultados de éstos con las predicciones, este ciclo de aprendizaje puede ser representado como PODS (Predicción-Observación-Discusión-Síntesis). Es así que un trabajo sostenido de investigación educativa en física, principalmente en la Universidad de Oregón, ha conducido al desarrollo de una metodología de enseñanza y aprendizaje las denominadas Clases Interactivas Demostrativas (Sokoloff \& Thornton, 2004).

Sokoloff propone un procedimiento de ocho pasos para las Clases Interactivas Demostrativas el cual retoman varios autores en América Latina para sus propias propuestas (Benegas, Alarcón y Zavála, 2013, Slisko y Medina-Hernández, 2007, Ramírez y Chávez, 2012) los cuales se presentan a continuación:

Clases Demostrativas Interactivas para la Formación de Profesores en línea. Mario Humberto Ramírez Díaz

Página 2 de 20 
1. El docente describe el experimento, si fuera necesario, lo realiza sin proyectar el resultado del experimento.

2. Los estudiantes deben de anotar su predicción individual en la Hoja de Predicciones, la cual será recogida al final de la clase, y donde el estudiante debe poner su nombre. Se debe asegurar a los estudiantes que estas predicciones no serán evaluadas, aunque una parte de la nota final del curso puede ser asignada por la simple asistencia a las Clases Interactivas Demostrativas.

3. Los estudiantes discuten sus predicciones en un pequeño grupo de discusión con los 2 o 3 compañeros más cercanos.

4. El docente obtiene las predicciones más comunes de toda la clase.

5. Los estudiantes registran la predicción final en la Hoja de Predicciones.

6. El docente realiza la demostración mostrando claramente los resultados.

7. Se pide a algunos estudiantes que describan los resultados, los discutan en el contexto de la demostración, anoten estos resultados en la Hoja de Resultados, la cual se llevan para estudiar.

8. Los estudiantes (o el docente) discuten situaciones físicas análogas con diferentes características superficiales (diferentes situaciones físicas) pero que responden al mismo concepto(s) físico.

\subsection{Formación de Profesores a Distancia.}

La educación tradicionalmente se ha apoyado en un modelo de enseñanza en el que, la exposición del profesor y la memorización de textos con el fin de presentar exámenes por parte del alumno son generalmente sus elementos principales. En este enfoque, el conocimiento es algo elaborado, propiedad del docente, el cual transmite a través de diferentes recursos a un grupo de alumnos que reciben pasivamente información y que compiten por obtener la atención del profesor, entre mayor atención lograda, se supone un mejor conocimiento (Blázquez, 2001, García 2010). El supuesto didáctico gira en torno a los elementos que permiten a un alumno no distraerse y enfocar su atención en el profesor, las clases son concebidas como espacios serios, silenciosos y con actividades individuales con interacción directa con el docente e indirecta con los mismos alumnos. Este modelo genera una serie de limitaciones que reducen la posibilidad de los alumnos para tener acceso a los mercados laborales en condiciones exitosas, incidiendo esto en su calidad y nivel de vida, ya que los saberes se circunscriben a lo que dicta el programa de estudios con pocas posibilidades de diversificarlo. Entre las limitaciones de la educación tradicional tenemos:

- $\quad$ Adquisición de datos e información a corto plazo.

- Habilidades limitadas a sus necesidades inmediatas no planificadas en el currículo.

Clases Demostrativas Interactivas para la Formación de Profesores en línea. Mario Humberto Ramírez Díaz

Página 3 de 20 
- $\quad$ Valores individualistas.

- Concepción limitada del trabajo en equipo.

- Dependencia hacia una figura de autoridad.

- $\quad$ Actitud pasiva hacia su formación académica.

Frente a esta problemática, la sociedad demanda una serie de características a los procesos educativos para que estos consigan superar sus limitaciones y logren impactar favorable y significativamente en la calidad de vida de la población. El Instituto Politécnico Nacional de México (IPN) responde a estas demandas a través de la construcción de un modelo educativo que ofrece satisfacción a las necesidades de formación de los niveles medio superior y superior con base en innovaciones educativas que brinden cobertura, flexibilidad, pertinencia, eficiencia y calidad para lograr una formación integral de los alumnos (IPN, 2004).

Por otro lado, la Comisión Internacional de Educación en Física (ICPE por sus siglas en inglés) la cual pertenece a la Unión Internacional de Física Pura y Aplicada (IUPAP), desde su creación en el año de 1960 ha reportado en diferentes escenarios (Ogborn, 2004), la gran problemática en la enseñanza y aprendizaje de la Física en todos los niveles, simplemente esto es reflejado por los elevados índices de reprobación en la materia (Black, Drake y Jossem, 2000, Gil y Vilches, 2009).

Por otro lado, México reiteradamente ha obtenido resultados no satisfactorios en las pruebas internacionales de aprovechamiento en ciencias que incluyen conceptos básicos de Física (INEE, 2013). Cabe señalar que la Asociación Internacional para la Evaluación del Logro Educativo (IEA) reveló que México había retirado los resultados correspondientes a las pruebas en Física de 1995 y 2000, debido a los bajos resultados obtenidos. En 2003 el Instituto Nacional para la Evaluación de la Educación (INEE) solicitó a la Dirección General de Educación de la SEP los resultados de las evaluaciones de 1995 y 2000. De esa forma se conocieron públicamente los resultados relacionados con la Física, la comprensión de información simple y compleja, la teorización, análisis y solución de problemas, el uso de herramientas, procedimientos de rutinas y procesos científicos, e investigación del mundo natural.

El profesor de Física se ubica en este complejo escenario educativo en el que cualquier decisión sobre la planeación didáctica del conocimiento tiene efectos importantes en la formación académica de sus alumnos. Se precisa entonces de una transposición didáctica del conocimiento teórico funcional que profesionalice entonces el conocimiento sobre el sistema escolar. Así, las acciones tendrían un sólido referente que, a su vez, permitan anticipar problemáticas y dificultades.

Las nuevas reformas educativas, así como el acceso a recursos tecnológicos para la educación ha modificado sustancialmente el escenario educativo. Resulta necesario que los profesores de Física accedan a sistemas de formación continua para mantenerse actualizados y que les permitan renovar su práctica docencia, adquiriendo conocimiento

Clases Demostrativas Interactivas para la Formación de Profesores en línea. Mario Humberto Ramírez Díaz

Página 4 de 20 
sobre los fundamentos y bases filosóficas de los enfoques de enseñanza y profundizando en las características y fundamentos de los diseños didácticos, uso de los recursos didácticos y herramientas tecnológicas. Ante esta realidad y necesidad de profesionalización docente, se hace patente un problema mayor: hacer compatible el interés de cursar un posgrado especializado con el tiempo dedicado al trabajo docente. Los programas de posgrado en la modalidad presencial y de tiempo completo exigen que cualquier postulante abandone su residencia y, en muchos de los casos, dejar la docencia. Para muchos profesores, los compromisos adquiridos con la institución educativa, con sus familias o sus proyectos personales limitan la posibilidad de estudiar un posgrado.

Para la consecución de las reformas de la propuesta del Modelo Educativo del IPN, es tarea indispensable de las escuelas centros y unidades del Instituto el desarrollo de escenarios innovadores de aprendizaje que reflejen su esencia, es decir una formación flexible, dinámica, integral, de alta calidad, centrada en el alumno, a partir de entornos de colaboración que le permitan al alumno abordar y proponer alternativas de solución a los problemas del entorno y así construir su aprendizaje. El Centro de Investigación en Ciencia Avanzada y Tecnología Aplicada Unidad Legaria del IPN (CICATA-IPN) es pionero en México y en Latinoamérica en la utilización de Ambientes Virtuales de Aprendizaje en programas de posgrado en Física Educativa.

Ante este contexto es que, en el 2005, año internacional de la física, el IPN propone la creación de un programa de posgrado en Física Educativa dirigido a atender las necesidades de formación para el gran sector de profesores de física a nivel nacional, tanto en su actualización profesional como en su formación como investigadores en el área. Para un profesor de Física, el programa representa el ingreso a una comunidad de colegas con preocupaciones comunes, problemáticas compartidas y experiencias diversas ${ }^{1}$. Este cúmulo de conocimiento empírico es una de las fuentes de conocimiento del cual se parte para transitar a una reflexión sistemática sobre los procesos educativos. Dado que se trata de profesores en activo, se tienen habilidades y conocimientos prácticos que son perfeccionados por los años de trabajo docente. Para realizar este proyecto académico, se emplea la plataforma tecnológica Moodle (open source) ${ }^{2}$, la cual está operando desde CICATA-IPN Unidad Legaria. En cada unidad de aprendizaje se deja explícito el tipo de interacción que se va a establecer, el tipo de productos que se van a obtener y finalmente el producto final que se va a generar. La gestión de los cursos se realiza con todos los profesores del núcleo básico, ya que se tiene el objetivo de retroalimentar el trabajo de los estudiantes de forma puntual. Los cursos son impartidos de forma modular, es decir, no se imparten de forma paralela. Se tiene la característica de que están articulados por temáticas, tipo de orientación, teórico o teórico-práctico, y por nivel de profundidad, básico, intermedio, avanzado, especializado.

Ante este panorama, en este trabajo se propuso la creación de una metodología basada en las CDI y que se pudiera implementar para la formación de profesores en la modalidad a distancia, teniendo como grupo de estudio el posgrado en Física Educativa del IPN, de

\footnotetext{
${ }^{1}$ http://www.cicata.ipn.mx/Paginas/Inicio.aspx

${ }^{2}$ Se puede consultar la plataforma en el siguiente sitio, sin embargo, solo los estudiantes y profesores del programa pueden accesar a los cursos regulares: http://www.fised.cicata.ipn.mx/
}

Clases Demostrativas Interactivas para la Formación de Profesores en línea. Mario Humberto Ramírez Díaz

Página 5 de 20 
manera particular con profesores de física en formación en el Doctorado en Física Educativa. En las siguientes secciones se detallarán las metas cumplidas y productos obtenidos en este trabajo.

\section{Metodología.}

En este trabajo se partió del siguiente objetivo general:

Estudiar el impacto de la implementación de metodologías alternativas en el aprendizaje de estudiantes de posgrado en línea.

Para el logro del anterior se propusieron los siguientes objetivos específicos:

1. Modificar las llamadas Clases Demostrativas Interactivas para ser implementadas en cursos a distancia.

2. Lograr un efecto multiplicador en los estudiantes de los profesores/estudiantes del curso de doctorado donde apliquen las Clases Demostrativas Interactivas para un tema en particular de física.

3. Obtener un portafolio de evidencias derivado de la aplicación de las CDI en los cursos de los profesores/estudiantes que permitan medir el impacto del uso de esta metodología de aprendizaje en educación a distancia.

4. Diseñar y aplicar una rúbrica de evaluación para medir el nivel de logro alcanzado por los estudiantes.

Por otro lado, la presente investigación siguiendo los enfoques de Hernández, Fernández y Baptista (2014, p. 385) y McMillan y Shumaher (2001) se inserta dentro del enfoque cualitativo de investigación. Lo anterior dado que el tema de investigación es del tipo evaluativo ya que se sitúa en una práctica concreta (en este caso la adaptación de las CDI en un ambiente a distancia) buscando evaluar sus méritos específicos, aplicada a una situación específica (estudiantes del programa de Doctorado en Física Educativa del IPN) y aumentar el conocimiento sobre el impacto de está metodología en el aprendizaje de profesores de física. Por lo tanto, este trabajo es del tipo investigación acción dado que el investigador fue también quien implemento la propuesta y la evaluó en el grupo seleccionado. De la misma forma, esta investigación es de tipo interactiva ya que, a pesar de ser con una muestra de sujetos a distancia, los instrumentos de análisis y la tecnología permiten un estudio a profundidad del fenómeno a partir de las experiencias de campo, tanto del investigador como de los sujetos de la muestra. También, esta investigación es un estudio de caso del tipo intrasituación dado que se limita al estudio de una sola entidad, el programa de doctorado en física educativa del IPN.

Con relación a la recolección de datos esta se hizo del tipo observación participante al ser una investigación de tipo interactiva ya que se usa una rúbrica (que se presenta más adelante) en la cual el papel del investigador en su evaluación juega un papel importante.

Clases Demostrativas Interactivas para la Formación de Profesores en línea. Mario Humberto Ramírez Díaz

Página 6 de 20 
La selección de muestra se realizo por medio de muestreo intencionado ya que se buscó aumentar la utilidad de la información obtenida a partir de pequeños grupos (McMillan y Schumaher, 2001, pp. 407). En este sentido la selección de escenario se dio con base en la propuesta de muestreo intencionado ya mencionada, de manera que la elección de sujetos de muestra permitiera obtener una diferencia máxima en las percepciones sobre el fenómeno a estudiar, de ahí que se eligiera el curso de Seminario de Investigación en Física Educativa II del Doctorado en Física Educativa del IPN dada la diferencia de perfiles de los 7 estudiantes inscritos, más adelante se hace una descripción detallada de este grupo muestra.

Para el cumplimiento de estos objetivos se platearon varias metas que se fueron alcanzando conforme se desarrolló la investigación las cuales se describen a continuación.

\subsection{Construcción de Clase Demostrativa Interactiva para Modalidad a Distancia}

Como se describió en la introducción del presente informe, las CDI son una metodología activa probada en la enseñanza de la física. Sin embargo, no existe una propuesta que implique su implementación en el aprendizaje en modalidad a distancia. Más aún, el autor original de la propuesta, David Sokoloff en entrevista realizada por el grupo de investigación en el marco de la Primera Escuela de Invierno en Física Educativa en CICATA de enero de 2016 mencionó: "Me parece difícil el introducir tecnología en la CDI ya que estas pueden perder su carácter interactivo y de describir fielmente a la naturaleza". Ante esta situación el reto fue, a partir del procedimiento de 8 pasos de las CDI hacer una adaptación que permitiera mantener en los estudiantes a distancia la interactividad además de seguir estudiando un fenómeno físico real a pesar de la interacción a través de medios tecnológicos, sin perder de vista que esta Clase Demostrativa Interactiva estaría dirigida a profesores de física. En este sentido, la implementación tal cual de los 8 pasos era inviable por lo que la propuesta de clase Demostrativa Interactiva a Distancia se construyó con la siguiente estructura:

1. Describir en plataforma un fenómeno físico y plantear la pregunta ¿Qué sucede?

2. Solicitar que se explique y redacte su opinión.

3. Pedir observar el fenómeno en video.

4. Contrastar su opinión con lo observado, ¿Corroboró o cambio su predicción?, Expliqué

5. ¿Cómo explicaría este fenómeno a nivel CONCEPTUAL a sus estudiantes?

6. Repita estos pasos con sus propios estudiantes y reporte sus resultados.

En el paso 6 se hace énfasis en el hecho de que los profesores pueden realizar una CDI "regular" al estar ellos en situación presencial (si el fenómeno a estudiar lo permite).

Clases Demostrativas Interactivas para la Formación de Profesores en línea. Mario Humberto Ramírez Díaz

Página 7 de 20 


\subsection{Selección de la muestra.}

Como se mencionó en la introducción y en los objetivos de este trabajo, el público objetivo son profesores en activo que se encuentran en realizando cursos de posgrado en el Programa de Posgrado de Física Educativa. Bajo esta óptica, se decidió diseñar e implementar una CDI a distancia siguiendo los 6 pasos propuestos en la sección anterior en el grupo de segundo semestre del Doctorado en Física Educativa en curso de Seminario de Investigación en Física Educativa II. Lo anterior como una de las actividades experimentales a desarrollar en el semestre enero-junio 2016. Esta muestra estuvo compuesta por 7 profesores estudiantes del programa de doctorado con el siguiente perfil:

1 Profesor de Ingeniería Industrial (México)

2 Profesores de Bachillerato Tecnológico (México)

1 Profesor de Ingeniería Civil y Pedagogía en Matemáticas (Chile)

1Profesor de Preescolar (México)

1 Profesor de Ingeniería (Colombia)

1 Profesor de Ingeniería (Ecuador)

Con este perfil de la muestra es interesante estudiar las diferentes respuestas hacia un mismo fenómeno a diferentes niveles y diferentes regiones en un solo grupo, situación que evidentemente es difícil de lograr en un grupo presencial.

\subsection{Fenómeno físico a estudiar.}

En este punto el grupo de la investigación se tenía que elegir un tema que fuera susceptible de ser estudiando para diferentes niveles ya que en el punto 6 de la propuesta se pide a los profesores que reproduzcan la CDI con sus propios estudiantes, lo cual hacia complejo que un fenómeno como la relatividad por ejemplo fuera difícil de implementar. En este sentido, se decidió estudiar el fenómeno de ingravidez al caer los cuerpos, y de manera específica el ejemplo de la caída de un reloj de arena. Esto implicó que el video grabara el fenómeno y se pusiera a disposición del usuario el video, esto se realizó mediante la grabación de la caída libre de un reloj de arena mediante una cámara Phantom (para tener la mejor calidad posible) y "subirlo" a YouTube ${ }^{3}$.

Como se comentó en los puntos anteriores, la CDI se implementó en el curso de Seminario de Investigación en Física Educativa II, el cual a su vez está disponible en la plataforma Moodle Institucional del IPN, de manera que esta fue una actividad regular del curso. Los estudiantes no sabían que estaban participando de una actividad de tipo experimental para este proyecto. Por parte del grupo de investigación se recolectó un portafolio de evidencias a partir de los materiales enviados por los profesores/estudiantes. En este portafolio de

\footnotetext{
${ }^{3}$ Disponible en https://www.youtube.com/watch?v=iXgotvplqUg
}

Clases Demostrativas Interactivas para la Formación de Profesores en línea. Mario Humberto Ramírez Díaz

Página 8 de 20 
evidencias en particular fue importante el material obtenido por los profesores al implementar en sus propios grupos la CDI, estas evidencias fueron audio, fotografías, reportes, hojas de predicción entre otros y se llevo un control y registro de estas en la rúbrica mostrada más adelante.

\section{Métodos y materiales.}

Como se mencionó en la sección anterior, se construyó una CDI destinada a ser implementada en la plataforma Moodle institucional en el curso de Seminario de Investigación Educativa II del Doctorado en Física Educativa, la pantalla de ingreso se muestra en la siguiente figura.

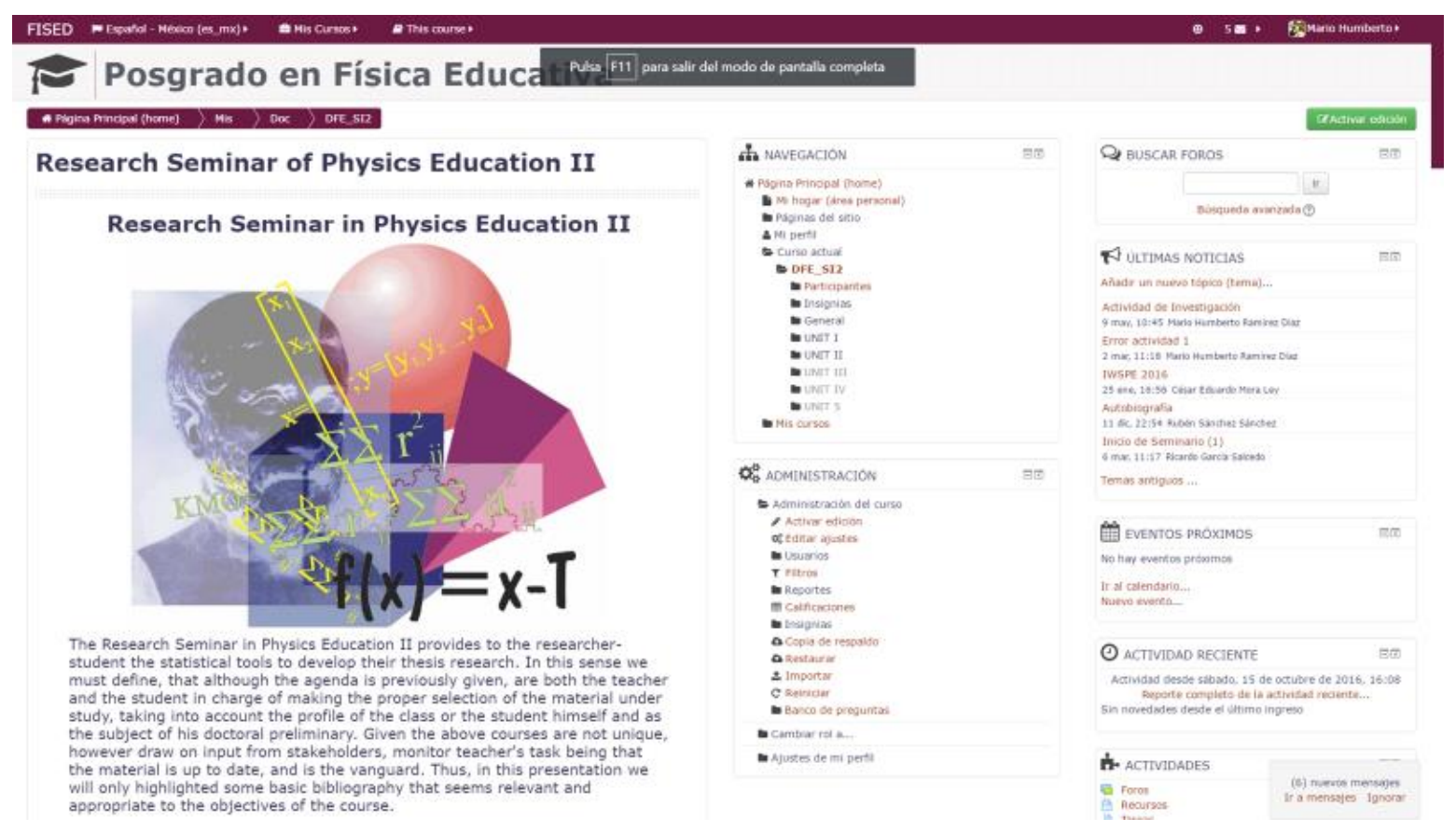

Figura 1. Pantalla de Ingreso al Curso de Seminario de Física Educativa II.

En este curso se implementó la CDI como actividad experimental al final de la unidad dos del curso, en la figura 2 se muestra la pantalla donde se implementa la actividad.

Clases Demostrativas Interactivas para la Formación de Profesores en línea. Mario Humberto Ramírez Díaz

Página 9 de 20 


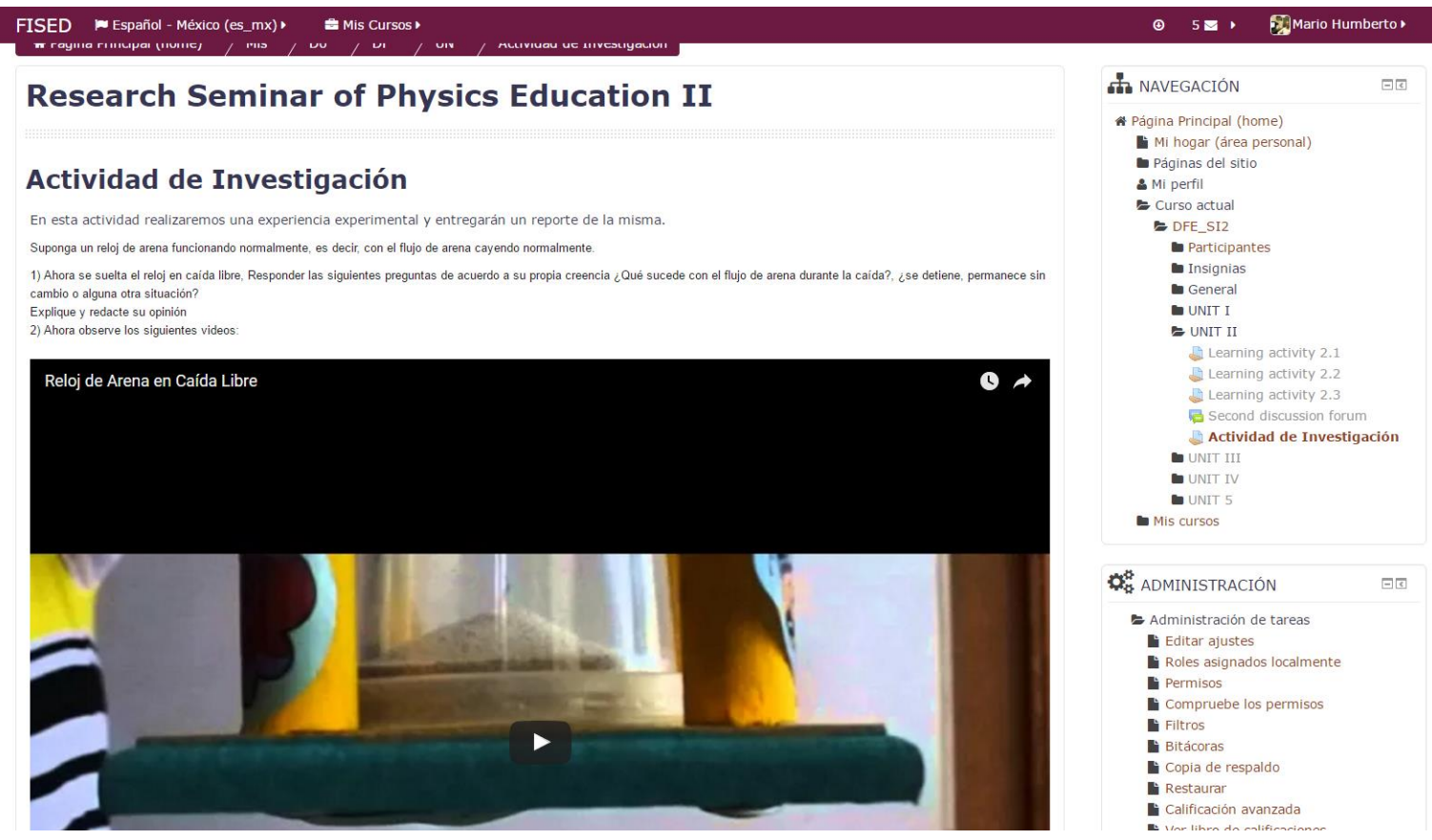

Figura 2. Pantalla de Ingreso a la actividad.

La CDI a detalle se presenta a continuación tal como les fue proporcionada a los participantes:

Actividad de Investigación

En esta actividad realizaremos una experiencia experimental y entregarán un reporte de la misma.

Suponga un reloj de arena funcionando normalmente, es decir, con el flujo de arena cayendo normalmente.

1) Ahora se suelta el reloj en caída libre, Responder las siguientes preguntas de acuerdo a su propia creencia ¿Qué sucede con el flujo de arena durante la caída?, ¿se detiene, permanece sin cambio o alguna otra situación? Explique y redacte su opinión

2) Ahora observe los siguientes videos

3) Contraste su opinión con lo observado, ¿Corroboró o cambio su predicción?, Expliqué

4) ¿Cómo explicaría este fenómeno a nivel CONCEPTUAL a sus estudiantes?

5) Repita estos pasos con sus propios estudiantes y reporte sus resultados.

Clases Demostrativas Interactivas para la Formación de Profesores en línea. Mario Humberto Ramírez Díaz

Página 10 de 20 
El video, que se encontraba insertado en la presentación de la actividad, fue el resultado de las video grabaciones del fenómeno descrita en la sección anterior. La dirección del video en YouTube es la siguiente:

\section{https://www.youtube.com/watch?v=jMFIZIqIgCY}

Como se mencionó en la sección anterior, los profesores/estudiantes conformaron un portafolio de evidencias como evaluación para su actividad, los resultados se mostrarán en la siguiente sección. Dicho portafolio de evidencias y el proceso de evaluación de la

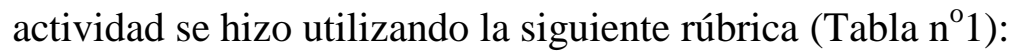

Tabla n ${ }^{0} 1$.

Rúbrica de evaluación para la CDI adaptada en línea (Creación propia)

\begin{tabular}{|c|c|c|c|}
\hline $\begin{array}{l}\text { Nivel de } \\
\text { logro }\end{array}$ & Básico & Medio & Alto \\
\hline $\begin{array}{l}\text { Da una explicación al } \\
\text { fenómeno previa a ver } \\
\text { el video }\end{array}$ & $\begin{array}{l}\text { No da una explicación } \\
\text { (correcta o incorrecta) } \\
\text { al fenómeno de } \\
\text { ingravidez. }\end{array}$ & $\begin{array}{l}\text { Hace una descripción } \\
\text { de su creencia de lo } \\
\text { que ocurre en el } \\
\text { fenómeno sin usar } \\
\text { términos y conceptos } \\
\text { formales de física. }\end{array}$ & $\begin{array}{l}\text { Da una explicación } \\
\text { del fenómeno en } \\
\text { términos y conceptos } \\
\text { formales usando } \\
\text { lenguaje científico. }\end{array}$ \\
\hline $\begin{array}{l}\text { Contrasta su creencia } \\
\text { previa con lo } \\
\text { observado en el video } \\
\text { y da conclusiones. }\end{array}$ & $\begin{array}{l}\text { No hace contrastación } \\
\text { de creencias previas, } \\
\text { solo describe lo } \\
\text { observado en el video. }\end{array}$ & $\begin{array}{l}\text { Hace contrastación de } \\
\text { creencias previas con } \\
\text { lo observado, pero no } \\
\text { llega a conclusiones } \\
\text { correctas o las no las } \\
\text { explica en términos de } \\
\text { lenguaje científico. }\end{array}$ & $\begin{array}{l}\text { Hace contratación de } \\
\text { creencias previas con } \\
\text { lo observado, llega a } \\
\text { una explicación del } \\
\text { fenómeno en términos } \\
\text { de ingravidez. } \\
\text { Revisa fuentes extra a } \\
\text { lo proporcionado en la } \\
\text { actividad. }\end{array}$ \\
\hline $\begin{array}{l}\text { Realiza el diseño de } \\
\text { una actividad dirigida } \\
\text { a sus estudiantes para } \\
\text { analizar el fenómeno }\end{array}$ & $\begin{array}{l}\text { No hace el diseño de } \\
\text { una secuencia } \\
\text { didáctica, a lo más } \\
\text { repite los pasos } \\
\text { proporcionados en la } \\
\text { actividad. }\end{array}$ & $\begin{array}{l}\text { Hace el diseño de una } \\
\text { secuencia didáctica } \\
\text { independiente de la } \\
\text { actividad llevada a } \\
\text { cabo en plataforma. }\end{array}$ & $\begin{array}{l}\text { Aplica la secuencia } \\
\text { didáctica diseñada a } \\
\text { grupos formales en la } \\
\text { clase de ciencias o } \\
\text { física y recopila } \\
\text { evidencias de la } \\
\text { aplicación. }\end{array}$ \\
\hline $\begin{array}{l}\text { Reporta la aplicación } \\
\text { de secuencia didáctica }\end{array}$ & $\begin{array}{l}\text { No presenta } \\
\text { resultados de la }\end{array}$ & $\begin{array}{l}\text { Presenta evidencias } \\
\text { de la aplicación de la }\end{array}$ & $\begin{array}{l}\text { Presenta portafolio de } \\
\text { evidencias de la }\end{array}$ \\
\hline
\end{tabular}

Clases Demostrativas Interactivas para la Formación de Profesores en línea. Mario Humberto Ramírez Díaz

Página 11 de 20 
RED. Revista de Educación a Distancia, Núm. 55, Artíc. 3

22-12-2017 DOI: http://dx.doi.org/10.6018/red/55/3 http://www.um.es/ead/red/55/ramirez.pdf

\begin{tabular}{|l|l|l|l|}
\hline $\begin{array}{l}\text { y los resultados de } \\
\text { esta en los } \\
\text { estudiantes. }\end{array}$ & $\begin{array}{l}\text { aplicación de } \\
\text { secuencia didáctica. }\end{array}$ & $\begin{array}{l}\text { secuencia didáctica en } \\
\text { los estudiantes, sin } \\
\text { embargo, no presenta } \\
\text { resultados del impacto } \\
\text { en el aprendizaje y } \\
\text { motivación de los } \\
\text { estudiantes. }\end{array}$ & $\begin{array}{l}\text { aplicación e impacto } \\
\text { de su propia secuencia } \\
\text { en los estudiantes, } \\
\text { mostrando } \\
\text { instrumentos o } \\
\text { procesos de } \\
\text { evaluación (diseñados } \\
\text { por el o retomados de } \\
\text { la literatura). }\end{array}$ \\
\hline
\end{tabular}

Es importante señalar que en este trabajo el objetivo es estudiar el impacto de la propuesta de las CDI adaptadas en el aprendizaje de los estudiantes del Doctorado en Física Educativa en relación con la propia aplicación de la metodología, siendo el tema propuesto la ingravidez un caso de estudio como se señalo anteriormente. Esto hace que la evaluación del impacto se haga (como se muestra en la rúbrica) acerca del seguimiento de las instrucciones en una primera etapa y en una segunda sobre la reproducción de la estrategia en los grupos a su vez de los estudiantes. Habitualmente, el proceso de evaluación de aprendizaje de las CDI se hace por medio de pre y post test (Sokoloff y Thorthon, 1998; Ramírez, Ortega y Ramírez, 2013) obteniendo ganancia conceptual a través de algún factor como el de Hake, sin embargo, en este trabajo dada la diferencia de perfiles de la muestra no es conveniente esta forma de evaluar dado que el objetivo final no es el aprendizaje del concepto de ingravidez sino lograr el desarrollo de habilidades de aprendizaje por parte del estudiante (de ahí la evaluación con rúbrica), para pasar de un nivel contemplativo a un nivel productivo de aprendizaje (Moltó, 2003).

\section{Resultados.}

En la sección III se comentó la importancia del perfil para esta primera implementación de la CDI en formato a distancia. Además, el portafolio de evidencias sería un buen parámetro del impacto (positivo o negativo) de la implementación de esta CDI.

En el caso del paso 1 de la CDI adaptada para ser implementada en línea, el fenómeno físico a estudiar -la ingravidez- se formuló en primera instancia como "experimento mental" el cual básicamente podía presentar 3 respuestas: a) No ocurre nada y sigue cayendo la arena; b) Se detiene el flujo de arena al comenzar a caer libremente el reloj, y c) La arena empieza a "flotar" como si empezara a fluir hacia arriba. Es importante señalar que el objetivo del programa de doctorado y el curso de Seminario de Física Educativa en general y de esta actividad en particular no es la física como disciplina, sino los procesos de aprendizaje de la física como disciplina dirigida a los diferentes nivel educativos; de tal forma que se hace énfasis en el carácter conceptual del problema, lo anterior debido a que podría pensarse en que este problema es de alta complejidad para un nivel preescolar por ejemplo o que se requiere del uso de ecuaciones complejas para poder resolverlo (lo cuál de hecho es verdad si se intenta simular matemáticamente).

Clases Demostrativas Interactivas para la Formación de Profesores en línea. Mario Humberto Ramírez Díaz

Página 12 de 20 
En su totalidad los estudiantes comentaron en su predicción que el flujo de arena se detiene, sin embargo, la explicación dada variaba entre el efecto anulado de la gravedad hasta el uso de analogías como es el caso de lo que sucede en un elevador. Después de ver el video los estudiantes corroboran el hecho de que el flujo de arena parece detenerse al empezar a caer el reloj de arena y de hecho empiezan a elaborar explicaciones dirigidas al nivel educativo donde enseñan, donde intervienen conceptos ya más formales como la fuerza de gravedad, la presión atmosférica e incluso el material que constituye la arena. Esto último es importante por qué, al ser un programa dirigido a la formación de profesores de física, lo más interesante de obtener es la trasposición didáctica que se podía lograr hacia los propios estudiantes de estos profesores/estudiantes, lo cual se explica en los resultados de los pasos 4 y 5 de la CDI modificada a continuación.

\subsection{Evidencias recolectadas.}

En el caso del estudiante colombiano, este implementó su propia secuencia en estudiantes de Ingeniería Civil de la Universidad Antonio Nariño, en Bogotá, Colombia. En este caso, el profesor decidió reproducir una CDI "tradicional" (como la descrita en la introducción de este trabajo). Como evidencia de la aplicación se enviaron vía la plataforma Moodle las hojas de predicción obtenidas, se muestra el ejemplo en la figura 3.

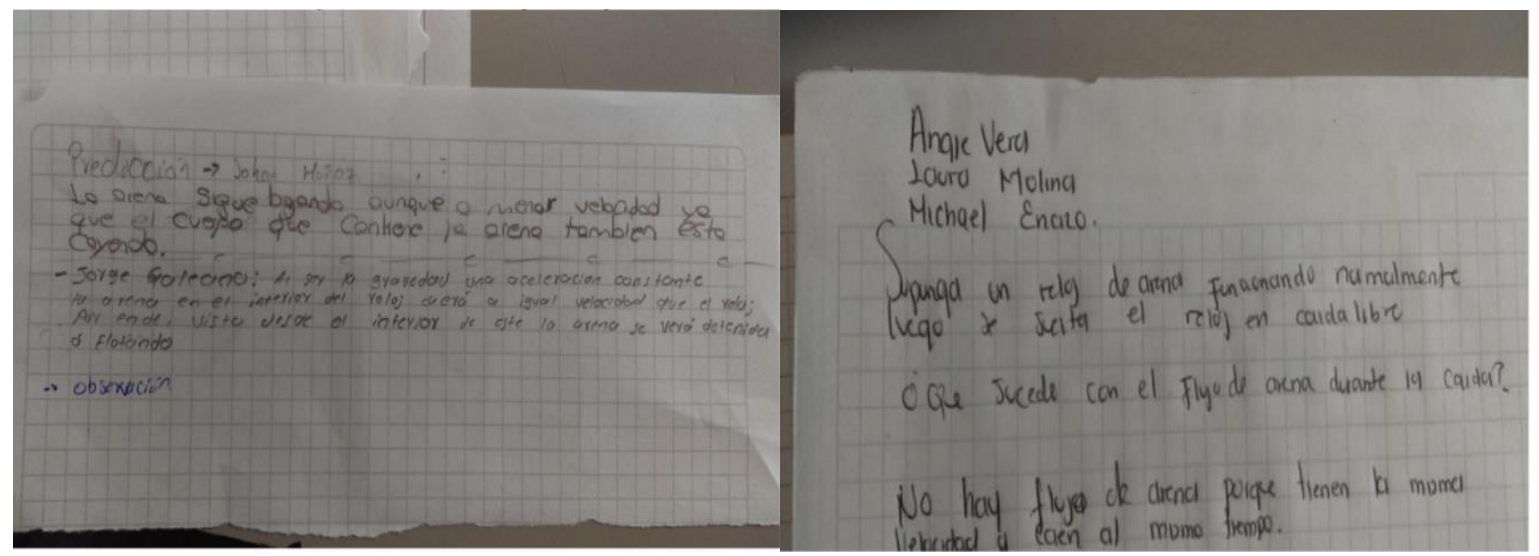

Figura 3. Evidencias de Aplicación de la CDI, Colombia.

En el caso del profesor de ecuatoriano, este implementó su secuencia en estudiantes de Ingeniería Civil en la Escuela Superior Politécnica del Litoral, en Guayaquil, Ecuador. En este caso, el profesor decidió introducir su propia plataforma (tipo Moodle) para que sus estudiantes estudiarán en el fenómeno, de manera que profundizaron más en situaciones como "el tipo de arena" para saber si el reloj de arena podría variar en función de ello, en la siguiente figura se muestra un ejemplo.

Clases Demostrativas Interactivas para la Formación de Profesores en línea. Mario Humberto Ramírez Díaz

Página 13 de 20 
El reloj de arena al hacerlo funcionar, el flujo de arena será constante durante su caída sin dejarlo caer todavía, en el momento que se deja caer el reloj de arena, en fracciones de segundos sufre un cambio de ordenamiento de los granos de arena. Los granos de arena siguen cayendo por efecto de la gravedad, y los granos de mayor tamaño y más denso caerán al fondo más rápido, mientras que los finos caerán después, este efecto no se ve, por motivo que estamos considerando una altura del reloj de unos $20 \mathrm{~cm}$ y de una alturade caída del reloj de arena unos $2 \mathrm{~m}$.

\begin{tabular}{|c|c|c|c|}
\hline Tamiz (ASTM) & $\begin{array}{c}\text { Tamiz (Neh) } \\
(\mathrm{mm})\end{array}$ & $\begin{array}{c}\text { Abertura real } \\
(\mathrm{mm})\end{array}$ & Tipo de suelo \\
\hline $3 "$ & 80 & 76,12 & \multirow{6}{*}{ GRAVA } \\
\hline $2=$ & 50 & 50,80 & \\
\hline $11 / 2 *$ & 40 & 38,10 & \\
\hline $1^{\prime \prime}$ & 25 & 25,40 & \\
\hline $3 / 4=$ & 20 & 19,05 & \\
\hline $3 / 8 "$ & 10 & 9,52 & \\
\hline$N^{\circ} 4$ & 5 & 4,76 & \multirow[t]{2}{*}{ \}ARENA GRUESA } \\
\hline $\mathrm{N}^{\circ} 10$ & 2 & 2,00 & \\
\hline $\mathrm{N}^{\circ} 20$ & 0,90 & 0,84 & \multirow[t]{3}{*}{ YARENA MEDIA } \\
\hline $\mathrm{N}^{\circ} 40$ & 0,50 & 0,42 & \\
\hline $\mathrm{N}^{\circ} 60$ & 0,30 & 0,25 & \\
\hline $\mathrm{N}^{\circ} 140$ & 0,10 & 0,105 & \multirow[t]{2}{*}{ ARENA FINA } \\
\hline $\mathrm{N}^{\bullet} 200$ & 0,08 & 0,074 & \\
\hline
\end{tabular}

Figura 1.5. Tabla de numeración y abertura de tamices. Fuente: Espinace R., 1979.

Figura 4. Evidencias de Aplicación de la CDI, Ecuador.

En el caso del profesor chileno, este implementó su secuencia en estudiantes de Pedagogía en Matemáticas de la Universidad Austral de Chile, en Puerto Montt, Chile. El profesor implemento una CDI en la que los estudiantes no solo escribían sus predicciones o conclusiones en las hojas, sino que se organizó una discusión acerca de lo que sucede en el fenómeno, el audio de estas discusiones fue grabado en audio para posterior análisis del profesor.

En el caso de la primera profesora mexicana, esta implementó su secuencia en estudiantes de Ingeniería Industrial de la Universidad Autónoma de san Luís Potosí. La profesora para hacer interactivo el experimento reprodujo su propia versión en un elevador y se hicieron mediciones, un ejemplo del reporte obtenido por los estudiantes y que sirvió como evidencia para la actividad se muestra en la siguiente figura.

Clases Demostrativas Interactivas para la Formación de Profesores en línea. Mario Humberto Ramírez Díaz

Página 14 de 20 


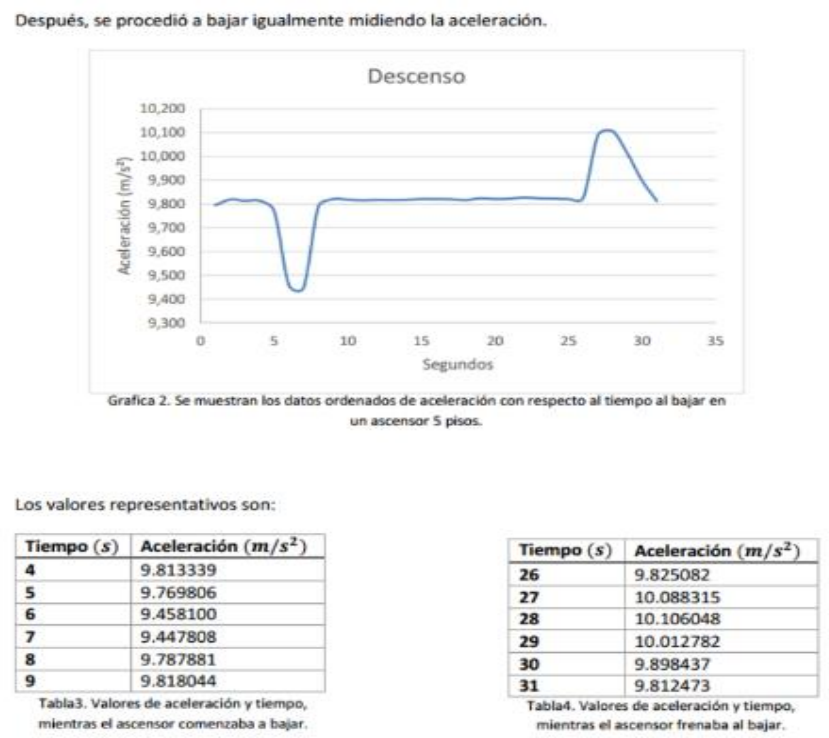

Figura 5. Evidencias de la Aplicación de la CDI, San Luís Potosí.

En el caso del segundo profesor mexicano, este aplicó su secuencia en estudiantes del Bachillerato Tecnológico en Cerámicos en la Preparatoria de Tonalá de la Universidad de Guadalajara. En este caso el profesor realizó un recuento de los comentarios realizados por los estudiantes, un extracto de dichos comentarios es la siguiente:

Los estudiantes, antes de ver el video, dieron respuestas como: a) no pasa nada, b) la arena baja más rápido, c) la arena se regresa. Es de destacar la respuesta tipo b), es curioso que crean que la arena caiga más rápido. En general no supieron dar las razones por las cuáles dieron sus opiniones. Hace falta que desarrollen la habilidad para explicar sus ideas, es posible también que haya sido por timidez o miedo a explicar su punto de vista. La calidad del video no deja apreciar claramente lo que sucede, sería necesario grabarlo con una mejor resolución. Es buen recurso para aplicar el ciclo PODS.

Finalmente, la última estudiante mexicana aplicó su secuencia didáctica en estudiantes de preescolar de un Colegio Privado en la Ciudad de México. En su caso la profesora implementó un ciclo de indagación en la que los pequeños construyeron su propio reloj de arena y vivieron el fenómeno al reproducirlo para reportar sus vivencias tanto oralmente como por medio de dibujos. Como evidencia de la experiencia se presentaron fotografías y videos del experimento, en la figura siguiente se muestran algunos ejemplos.

Clases Demostrativas Interactivas para la Formación de Profesores en línea. Mario Humberto Ramírez Díaz

Página 15 de 20 


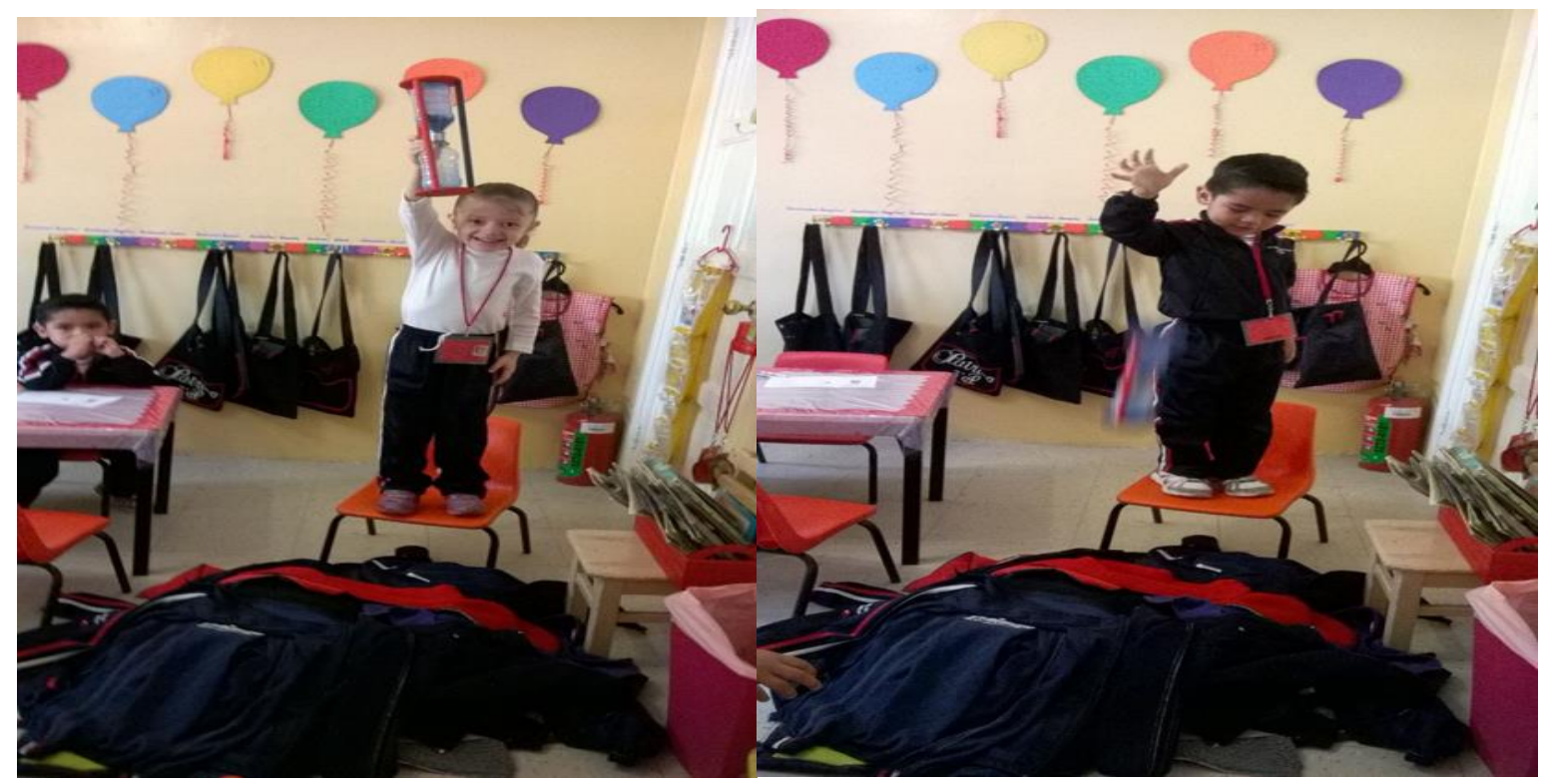

Figura 6. Evidencias de la Aplicación de la CDI, Preescolar.

Los resultados mostrados fueron evaluados a partir de la rúbrica expuesta en las secciones anteriores e indican en los siete casos un nivel de logro alto obtenido. En todos los casos, los estudiantes dieron explicaciones al fenómeno de la ingravidez comparados con su predicción, además de compartir en los foros de la plataforma sus propias explicaciones y posteriores conclusiones. Por otro lado, los siete estudiantes crearon secuencias didácticas en las cuales incorporaron elementos de la metodología CDI, recopilando también portafolio de evidencias. Comparando las evidencias es notorio que, en los cursos regulares donde se implemento la actividad por parte de los estudiantes se obtuvieron resultados positivos llevando también a un alto nivel de logro. Al igual que en la actividad en plataforma, ninguno de los estudiantes aplico el procedimiento de pre y post test a pesar de que en 6 de los siete casos se implemento en la clase de física y la otra en clase de ciencias (en preescolar). Sin embargo, en todos los casos obtuvieron conclusiones que cambiaban la predicción hecha antes de ver el video o experimentar directamente el fenómeno, la cuál resulto correcta en los términos conceptuales de la ingravidez.

Después de esta primera aplicación se obtuvieron conclusiones interesantes para hacer correcciones en nuevas implementaciones. De hecho, se ha podido implementar esta metodología en (ya no como grupo de investigación) en la Maestría en Física Educativa en el curso de Metodología de la Investigación Educativa II en el semestre junio-diciembre 2016, y en cursos de formación de profesores de nivel bachillerato en el estado de Oaxaca, México organizados por el Centro Interdisciplinario de Investigación para el Desarrollo Regional del IPN en enero de 2017.

Clases Demostrativas Interactivas para la Formación de Profesores en línea. Mario Humberto Ramírez Díaz

Página 16 de 20 


\section{Conclusiones e impacto de la investigación.}

Como resultado principal de esta investigación se puede concluir que -al menos en el caso de la formación de profesores de física- es posible implementar Clases Demostrativas Interactivas en modalidad a distancia, lo cual es un aporte a la disciplina, en la cual derivado de los trabajos de Sokoloff no se considera que las CDI se pudieran implementar por medio de tecnología.

Una de las ventajas principales es la interacción de profesores de diferentes niveles sobre un mismo tema, en este caso de física, ya que se pueden intercambiar estrategias y retroalimentaciones obtenidas por los estudiantes, de manera que se enriquece la práctica, situación que es posible en la plataforma Moodle por medio de los foros -de manera formal- y en las redes sociales y de manera personal -de manera informal-.

Parte de esta interacción es también el intercambio entre diferentes regiones, en este trabajo se tuvo intercambio entre profesores de México, Colombia, Ecuador y Chile, situación que evidentemente no es posible en las CDI regulares que se implementan de manera presencial.

Las evidencias muestran un beneficio no solo en la formación del profesor sino en el aprendizaje de sus estudiantes (objetivo último evidentemente), ya que el portafolio de evidencias recolectado por los profesores y que a su vez era parte del punto 5 de la propuesta implementada (y que parte de estas fueron mostradas en la sección anterior). Los profesores participantes como parte de su propia implementación desarrollaron estrategias de evaluación del aprendizaje, las cuales a su vez mostraron un avance en sus estudiantes, dependiendo del nivel y región donde se implementó la CDI. La situación anterior refuerza la conclusión de la eficiencia de la propuesta implementada derivada de este proyecto de investigación.

Sin embargo, la propuesta tiene también algunas limitaciones, entre estas la falta de interacción síncrona entre los participantes puede representar una debilidad en la implementación y que a pesar de que, si hubo interacción entre los participantes vía los foros de asesoría y chats de la plataforma Moodle institucional, esta no es en tiempo real y el retraso en la comunicación puede llevar a que no se puedan hacer correcciones en las propuestas de los compañeros. Otra limitación es el hecho de que no cualquier tema de física es viable de implementar como CDI en línea, sobre todo dado que en un curso regular de formación de profesores se puede tener (como fue el caso de la implementación reportada en las secciones anteriores) una diversidad de profesores de diferentes niveles y regiones, que trabajen en diferentes sistemas y que no necesariamente sean compatibles de llevar a cabo en la práctica.

Presentación del artículo: 12 de mayo de 2017

Fecha de aprobación: 12 de diciembre de 2017

Clases Demostrativas Interactivas para la Formación de Profesores en línea. Mario Humberto Ramírez Díaz

Página 17 de 20 
Ramírez, M. (2017). Clases Demostrativas Interactivas para la formación de profesores en línea. Revista de Educación a Distancia, 55. Consultado el (dd/mm/aaaa) en http://www.um.es/ead/red/55/ramirez.pdf

Fecha de publicación: 22 de diciembre de 2017

\section{FINANCIACIÓN}

El presente trabajo se inserta en el proyecto de investigación denominado "Estudio del impacto de secuencias de aprendizaje activo de la física en estudiantes de posgrado en línea" (SIP20164794).

\section{Referencias Bibliográficas.}

Benegas, J., Alarcón, H. y Zavala, G. (2013). Formación de Profesorado en Metodologías de Aprendizaje Activo de la Física. En J. Benegas, M. C. Pérez de Landazabal y J. Otero (Eds.). El Aprendizaje Activo de la Física Básica Universitaria (pp. 193-203). Santiago de Compostela, España: Andavira.

Black, P. (2000). Physics Education in the New Millenium. En Black, P., Drake, G. $y$ Jossem, L. (Eds.)

Blázquez, F. (Coordinador)(2001). Sociedad de la información y Educación. Junta De Extremadura, Consejería de Educación, Ciencia y Tecnología Dirección General de Ordenación, Renovación y Centros Mérida.

Briscoe, C. (1991). The dynamic interactions among beliefs, role methaphores and teaching practices. A case study of teacher change. Science Education, 75(2), 185-99.

Bromme R(1988) .Conocimientos profesionales de los profesores. Enseñanza de las Ciencias 6 (1), 19-29.

Cronin-Jones, L.L (1991). Science teaching beliefs and their influence on curriculum implementation: two case studies. Journal of Research in Science Teaching, 38 (3), $235-50$.

García, L. (2010). ¿Se sigue dudando de la educación a distancia? REOP. 21 (2), 240-250.

Gil, D. y Vilches, A. (2009). Formación del profesorado. Reflexiones de profesionales de la educación, OEI, Metas Educativas 2021.

Clases Demostrativas Interactivas para la Formación de Profesores en línea. Mario Humberto Ramírez Díaz

Página 18 de 20 
Hernández, R., Fernández, C. y Baptista, M. (2014). Metodología de Investigación, McGraw-Hill/Interamericana Editores, México.

Hernández, C., Tecpan, S. y Osorio, A. (2015). Aprendizaje Activo Para Futuros Docentes De Física: Estrategias En Un Curso De Didáctica. Actas IV Jornadas de Enseñanza e Investigación Educativa en el campo de las Ciencias Exactas y Naturales Facultad de Humanidades y Ciencias de la Educación. Universidad Nacional de La Plata.

INEE. (2013). http://www.inee.edu.mx/index.php/publicaciones/resultados-deinvestigacion/cuadernos-de-investigacion/81-publicaciones/uadernos-deinvestigacion-capitulos/549-4-tercer-estudio-internacional-de-matematicas-yciencias-naturales-timss-resultados-de-mexico. Consultado el 12 de septiembre de 2016.

INEGI.(2013). México en cifras, http://www.inegi.org.mx/sistemas/mexicocifras/default.aspx , consultado el 10 de septiembre de 2016.

IPN. (2004). Materiales para la reforma Libro 1: Un Nuevo modelo educativo para el IPN, Instituto Politécnico Nacional, México.

McDermott, L. (1984). Research on conceptual understanding in mechanics. Physics Today 37, 24-32.

McMillan, J. y Schumaher, S. (2001). Investigación Educativa, Una introducción conceptual. Pearson Addison Wesley, Madrid, España.

Moltó, E. (2003). Fundamentos Psicológicos de La enseñanza y el Aprendizaje. Ministerio de Educación, La Habana, Cuba.

Ogborn, J. (2004), Physics now, I.U.P.A.P.-39, London. Disponible en http://web.phys.ksu.edu/ICPE/Publications/PhysicsNowText-A4.pdf

Ramírez, M. y Chávez, E. (2012). Similitudes Del Sistema 4MAT De Estilos De Aprendizaje Y La Metodología De Clases Interactivas Demostrativas En La Enseñanza De La Física. Revista Estilos de Aprendizaje, 9, (9), 141-155.

Ramírez, M., Méndez-Sánchez, A., Pérez-Trejo, L. y Olvera, M. (2016). Competencias específicas consideradas más realizadas y más importantes en los programas de física en México. Perfiles Educativos, 38 (152), 60-87.

Ramírez, Y., Ortega, A. y Ramírez, M. (2013). Clases demostrativas interactivas de magnetismo en el bachillerato del IPN. Lat. Am. J. Phys. Educ. 7 (1), 28-36.

Clases Demostrativas Interactivas para la Formación de Profesores en línea. Mario Humberto Ramírez Díaz

Página 19 de 20 
Slisko, J. y Medina-Hernández, R. (2007). Un curso de mecánica clásica sin conferencias magisteriales: objetivos, elementos del diseño y efectos en los estudiantes, Lat. Am. J. Phys. Educ. 1 (1), 51-61.

Sokoloff, D. (2006). Aprendizaje Activo de Óptica y Fotónica, Manual de Entrenamiento ALOP.UNESCO.

Sokoloff, D. \& Thornton. R. (2004). Interactive Lecture Demostrations, Active Learning in Introductory Physics. John Wiley \& Sons, Inc.

Sokoloff, D. \& Thornton, R. (1998). Assessing student learning of Newton's laws: The Force and Motion Conceptual Evaluation and the evaluation of active learning laboratory and lecture curricula. Am. J. Phys. 66, 338-352.

Clases Demostrativas Interactivas para la Formación de Profesores en línea. Mario Humberto Ramírez Díaz

Página 20 de 20 